\title{
An analysis of satellite calibration methods for CCD astrometry of Saturn's satellites
}

\author{
K. X. Shen ${ }^{1,2,3}$, G. Dourneau ${ }^{4}$, R. C. Qiao ${ }^{1,3}$, and J. R. Liu ${ }^{1,3}$ \\ 1 Shannxi Astronomical Observatory, Lintong, Shannxi 710600, PR China \\ 2 Chinese National Astronomical Research Center \\ 3 United Laboratory for Optical Astrometry, The Chinese Academy of Science, PR China \\ 4 Observatoire de l'Université Bordeaux I, BP 89, 33270 Floirac, France
}

Received 25 July 2000 / Accepted 28 November 2000

\begin{abstract}
This paper presents an analysis of astrometric reduction methods for the calibration of a CCD target. To compare these methods, we collected about 3000 recent CCD observations between 1990-1997. We discuss the comparison of the four main contemporary theories describing the eight major satellites of Saturn, used in recent CCD astrometric reduction. We show that these theories developed by Taylor \& Shen (1988), Dourneau (1993), Harper \& Taylor (1999) and Duriez \& Vienne (1997), give a rather good representation of the orbits of the eight main satellites, especially for satellites III-VI. In the CCD astrometric reduction, we point out a bias of the theories on the derived satellite positions, which can reach about 0.'03. Duriez and Vienne's TASS theory (1997), built with significantly higher consistency than the other three, generally leads to the lowest residuals for the observations analysed here. Due to its high quality, we recommend use of this theory for CCD reduction. Systematic errors affecting satellites' derived positions should be small, due to the quality of the TASS theory. This procedure might be an alternative to the multi-theory reduction method previously proposed by Qiao et al. (1999). Observations of satellites obtained from CCD reduction using TASS are expected to be significantly more accurate $\left(0.0^{\prime \prime} 015\right)$ than observations reduced from any other theory (about $0 . \prime 05$ ).
\end{abstract}

Key words. planetes \& satellites: Saturn - astrometry - methods: observational

\section{Introduction}

The CCD technique in astrometry, which has been used for about a decade, has some intrinsic difficulties in dealing with astrometric reduction. This is a consequence of the small size of the CCD target, generally associated with the use of a long-focus telescope. In order to obtain profitable data, we must know precisely the focal length of the telescope and the orientation of the target at each observation. In recent years, much effort has been devoted to finding more reliable methods of calibration. Jones et al. (1989) used the wide double star 61 Cygni to determine the calibration parameters of CCD exposures. Colas \& Arlot (1991) chose to use globular clusters such as M 15, for which astrometric catalog data are available. Neither of these methods are quite satisfactory from an astrometric perspective. So, other methods were proposed, using the bright moons of Saturn, whose positions are derived from well-known ephemeris data (Harper et al. 1997). Such methods have already been used in some previous astrophotographic observations (Veillet \& Dourneau 1982), but present a problem because errors of satellite

Send offprint requests to: G. Dourneau, e-mail: dourneau@observ.u-bordeaux.fr ephemerides affect their derived positions (Pascu et al. 1987). In the present paper, we confirm this error from our analysis of observations. In order to reduce systematic errors which affect satellite observations reduced from the use of only one theory, Qiao et al. (1999) proposed the multi-theory method. This method uses the four contemporary dynamical satellite theories developed by Taylor \& Shen (1988), Dourneau (1993), Harper \& Taylor (1993) and Duriez \& Vienne (1997). Satisfactory observations of satellites were provided by the multi-theory method, providing observed-minus-computed residuals which can reach 0 !'08. In this paper, we will analyze the accuracy of various CCD reduction methods based on the four satellite theories, including the multi-theory method that we will use as a reference for comparison. From a detailed analysis of these theories, we will try to suggest a CCD reduction method determining more accurately the positions of saturnian satellites.

\section{Theories and observations used in the analysis}

\subsection{Theories}

The theories that we will analyze in this paper are the four main contemporary theories mentioned above. 
Three of these theories, developed by Dourneau (1993), Taylor \& Shen (1988) and Harper \& Taylor (1997) are constructed from previous theories developed by Struve (1898) and later improved by Struve (1931) and Kozai (1957). The differences between these three theories are in the quantity and the quality of observations used to adjust the theoretical constants. Also, the number of adjusted constants and the accuracy of the adjustment method significantly differs between theories. For example, Taylor \& Shen (1988) used photographic observations over the 1967-1983 period to determine a limited number of satellite orbital elements. Dourneau (1987) corrected more than a hundred theoretical constants relative to the eight major saturnian satellites from a century of observations, including all published photographic and visual micrometric data from 1886 to 1985 . In this paper, we used the last version of this theory, in which Dourneau (1993) corrected some more libration parameters of the four inner satellites. This new version recently allowed a determination of the masses of these satellites (Dourneau \& Baratchart 1999). Harper \& Taylor also corrected a great number of constants of the major saturnian satellites. They referred to the Strugnell \& Taylor (1990) catalog of satellite observations from 1874 to 1989, including Pascu's unpublished (1982) observations made at U.S. Naval Observatory. The new version of this theory (Harper \& Taylor 1999), used in our paper, is fitted to some additional recent CCD observations from 1990 to 1997 for all satellites, Hyperion excepted. For Hyperion, this new version is derived from the synthetic theory developed by Taylor (1992). It includes additional terms for the perturbations of Titan and is based on observations from 1967 to 1982. From this analysis, we can expect Dourneau's and Harper \& Taylor's theories to be rather similar, except for Hyperion. Also, both of these theories, based on a long period of observation, should be significantly more reliable than Taylor \& Shen's, adjusted to a limited collection of observed data. TASS theory (Théorie Analytique des Satellites de Saturne) has been presented in various successive papers by Vienne \& Duriez (1991, 1992, 1995). They propose new analytical expressions for orbital elements, including additional perturbations of satellites. TASS has a very good internal consistency of a few kilometers, significantly better than the other three, more classical, contemporary theories. Shen \& Qiao (1996) have shown that TASS accuracy is now greater than the precision of photographic observations. In this paper, we have used the newest TASS 1.7 version (Duriez \& Vienne 1997) including Hyperion and adjusted to the observed data of the Strugnell \& Taylor (1990) catalog. The four contemporary theories will be compared further in this paper to the observations that we have collected and that we will present now.

\subsection{Observations}

In this work, we have collected about 3000 CCD observations of Saturn's satellite positions. The first 500 of them were reported in our previous paper (Qiao et al. 1999). They were made in the period 1994-1996 with the $1.56 \mathrm{~m}$ telescope at the Sheshan Station near Shanghai, China. The approximate focal length of the telescope was about $15.6 \mathrm{~m}$ and the size of the square pixels of the CCD cell was $22 \times 22 \mu \mathrm{m}$. In addition, we collected another 2500 CCD positions, successively obtained from 1990 to 1997 by Beurle et al. (1993) and Harper et al. (1997, 1999), with the $1 \mathrm{~m}$ Jacobus Kapteyn telescope on the island of La Palma, Spain. All these observations are gathered in 21 datasets listed in Table 1. The first column gives names of datasets in which $\mathrm{H}$ and $\mathrm{Q}$, respectively, denote Harper and Qiao's observations. For each dataset, Cols. 7-9 specify how many times each calibration satellite, Tethys, Dione and Rhea, was observed. Titan, used as a reference satellite, is not specified, but the number of its observations is the total of all mentioned calibration satellite numbers. The last column indicates the number of nights of observation for each dataset. Qiao's datasets always involve only one night, while Harper datasets can involve several nights. However, during each dataset, the CCD camera was kept fixed on the telescope, without any manipulation of the target.

\section{CCD astrometric reduction method}

\subsection{Computation of calibration parameters}

The images of satellites on CCD frames provide raw measured intersatellite rectangular coordinates $\Delta x$ and $\Delta y$ of the observed satellite $\mathrm{B}$ relative to the reference satellite A. From these rectangular coordinates, measured in pixels, we can derive other measured coordinates, such as position angle $P_{\mathrm{m}}$ and separation $S_{\mathrm{m}}$, commonly associated with old micrometric observations.

$P_{\mathrm{m}}=\arctan (\Delta x / \Delta y)$

$S_{\mathrm{m}}=\left(\Delta x^{2}+\Delta y^{2}\right)^{1 / 2}$

$P_{\mathrm{m}}$ refers to the vertical axis of the CCD frame, and $S_{\mathrm{m}}$ is expressed in pixels. From such raw data, we can derive observed coordinates to be compared to computed coordinates, such as position angle $P$, referred to the true pole of date, and separation $S$, expressed in arc seconds, by the following relations:

$P=P_{\mathrm{m}}+\delta P$

$S=k S_{\mathrm{m}}$

in which $\delta P$ and $k$ are defined as calibration parameters of CCD exposures. $k$ represents the scale of the CCD frame, expressed in arcsecond per pixel. $\delta P$ is the correction to the position angle of the true pole of date with respect to the north-south axis of the CCD array, measured positive towards the east. Observed coordinates $P$ and $S$ can be compared to topocentric computed coordinates $P_{\mathrm{c}}$ and $S_{\mathrm{c}}$, expressed in the true equator and equinox of date. We applied to these computed coordinates corrections for 
Table 1. Collected datasets of Saturn's satellite CCD observations, denoted by H and Q respectively for Harper et al. (1997, 1999) and Qiao et al. (1999) observations. Values of the calibration parameters computed in this paper from the four contemporary satellite theories are given and can be compared with previous values adopted by the authors. Respective numbers of calibration satellites and nights of observation are specified for each dataset

\begin{tabular}{|c|c|c|c|c|c|c|c|c|c|}
\hline \multirow[t]{3}{*}{ Datasets } & \multicolumn{5}{|c|}{ Calibration parameters } & \multicolumn{3}{|c|}{ Number of } & \multirow{3}{*}{$\begin{array}{l}\text { Number of } \\
\text { nights of } \\
\text { observation }\end{array}$} \\
\hline & T\&S & $\mathrm{H} \& \mathrm{~T}$ & TASS & Dourneau & Adopted & calibr: & ion sat & llites & \\
\hline & \multicolumn{4}{|c|}{ above: $\delta P(\circ)$ below: $k\left({ }^{\prime \prime} /\right.$ pixel $)$} & Values & Tethys & Dione & Rhea & \\
\hline \multirow[t]{2}{*}{ H90A } & 1.1624 & 1.1729 & 1.1711 & 1.1802 & 1.1746 & 40 & 31 & 47 & 9 \\
\hline & 0.302802 & 0.302871 & 0.302820 & 0.302764 & 0.302844 & & & & \\
\hline \multirow[t]{2}{*}{ H90B } & 1.2056 & 1.2165 & 1.2104 & 1.2255 & 1.2089 & 6 & 6 & 6 & 7 \\
\hline & 0.302907 & 0.302950 & 0.302877 & 0.302843 & 0.302802 & & & & \\
\hline \multirow[t]{2}{*}{ H90C } & 1.1050 & 1.1075 & 1.1015 & 1.1191 & 1.0900 & 1 & 2 & 3 & 2 \\
\hline & 0.302882 & 0.302845 & 0.302796 & 0.302706 & 0.302902 & & & & \\
\hline \multirow[t]{2}{*}{ H91 } & 0.2403 & 0.2739 & 0.2660 & 0.3035 & 0.2682 & 18 & 17 & 18 & 6 \\
\hline & 0.302945 & 0.303078 & 0.302975 & 0.302956 & 0.302936 & & & & \\
\hline \multirow[t]{2}{*}{ H93 } & 2.4404 & 2.4513 & 2.4503 & 2.4636 & 2.4525 & 28 & 30 & 30 & 7 \\
\hline & 0.309729 & 0.309822 & 0.309733 & 0.309747 & 0.309693 & & & & \\
\hline \multirow[t]{2}{*}{ H94 } & 0.0954 & 0.0952 & 0.0948 & 0.1077 & 0.0959 & 12 & 19 & 20 & 2 \\
\hline & 0.309801 & 0.309910 & 0.309762 & 0.309810 & 0.309801 & & & & \\
\hline \multirow[t]{2}{*}{ Q940811 } & -5.4787 & -5.6171 & -5.5880 & -5.7072 & -5.4898 & 0 & 4 & 16 & 1 \\
\hline & 0.249382 & 0.250093 & 0.249974 & 0.250128 & 0.249900 & & & & \\
\hline \multirow[t]{2}{*}{ Q940813 } & -5.5157 & -5.5407 & -5.5625 & -5.5639 & -5.5750 & 15 & 39 & 39 & 1 \\
\hline & 0.249335 & 0.249578 & 0.249663 & 0.249645 & 0.249400 & & & & \\
\hline \multirow[t]{2}{*}{ Q941022 } & 2.1273 & 2.1019 & 2.0945 & 2.0842 & 2.1540 & 4 & 23 & 23 & 1 \\
\hline & 0.249917 & 0.249936 & 0.249936 & 0.249832 & 0.249713 & & & & \\
\hline \multirow[t]{2}{*}{ Q941023 } & 2.2191 & 2.1866 & 2.2012 & 2.1832 & 2.2064 & 14 & 14 & 14 & 1 \\
\hline & 0.250023 & 0.250290 & 0.250027 & 0.250204 & 0.249857 & & & & \\
\hline \multirow[t]{2}{*}{ Q941024 } & 1.4426 & 1.4362 & 1.4396 & 1.4192 & 1.4520 & 5 & 0 & 5 & 1 \\
\hline & 0.249697 & 0.249931 & 0.249820 & 0.249882 & 0.249850 & & & & \\
\hline \multirow[t]{2}{*}{ Q941025 } & 2.1946 & 2.1938 & 2.1906 & 2.1815 & 2.2044 & 6 & 6 & 6 & 1 \\
\hline & 0.249869 & 0.249954 & 0.249827 & 0.249868 & 0.249820 & & & & \\
\hline \multirow[t]{2}{*}{ H95 } & 0.1328 & 0.1533 & 0.1464 & 0. 1562 & 0.1529 & 36 & 42 & 56 & 13 \\
\hline & 0.330323 & 0.330431 & 0.330322 & 0.330340 & 0.330352 & & & & \\
\hline \multirow[t]{2}{*}{ Q950815 } & 2.2064 & 2.2383 & 2.2054 & 2.2062 & 2.2485 & 9 & 19 & 20 & 1 \\
\hline & 0.249034 & 0.250035 & 0.250272 & 0.250293 & 0.249800 & & & & \\
\hline \multirow[t]{2}{*}{ Q950816 } & 2.2625 & 2.2675 & 2.2622 & 2.2516 & 2.2585 & 0 & 7 & 7 & 1 \\
\hline & 0.249838 & 0.250082 & 0.249998 & 0.250055 & 0.249850 & & & & \\
\hline \multirow[t]{2}{*}{ Q950922 } & 2.3784 & 2.1750 & 2.2882 & 2.1338 & 2.2450 & 3 & 9 & 9 & 1 \\
\hline & 0.249881 & 0.249893 & 0.249785 & 0.249798 & 0.249950 & & & & \\
\hline \multirow[t]{2}{*}{ Q961018 } & 7.6132 & 7.7188 & 7.7433 & 7.7602 & 7.5142 & 12 & 12 & 0 & 1 \\
\hline & 0.252047 & 0.251500 & 0.251384 & 0.251383 & 0.251170 & & & & \\
\hline Q961019 & 7.4846 & 7.4885 & 7.4920 & 7.4787 & 7.4782 & 13 & 14 & 13 & 1 \\
\hline & 0.251185 & 0.251469 & 0.251381 & 0.251522 & 0.251275 & & & & \\
\hline H97A & 0.5764 & 0.5789 & 0.5663 & 0.5438 & 0.5684 & 89 & 42 & 84 & 1 \\
\hline & 0.330173 & 0.330407 & 0.330346 & 0.330497 & 0.330407 & & & & \\
\hline H97B & 0.6159 & 0.5871 & 0.6040 & 0.5814 & 0.6126 & 68 & 68 & 61 & 1 \\
\hline & 0.330227 & 0.330497 & 0.330383 & 0.330560 & 0.330389 & & & & \\
\hline H97C & 0.6544 & 0.6437 & 0.6502 & 0.6502 & 0.6449 & 35 & 35 & 26 & 1 \\
\hline & 0.330210 & 0.330435 & 0.330349 & 0.330418 & 0.330349 & & & & \\
\hline
\end{tabular}

light-time, refraction and stellar aberration. Both calibration parameters $k$ and $\delta P$ are determined in the reduction process from their initial values $k_{0}$ and $\delta P_{0} . k_{0}$ is evaluated from the approximate value of the focal length of the telescope, and $\delta P_{0}$ from some index points marked on the telescope and on the CCD device, so that:

$k=k_{0}+\mathrm{d} k$
$\delta P=\delta P_{0}+\mathrm{d} P$. $\mathrm{d} k$ and $\mathrm{d} P$ are corrections to initial values derived from the adjustment between observed and computed satellite positions. This adjustment consists of minimizing residuals between observed and computed coordinates:

$\Delta S=S-S_{\mathrm{c}}$ 
Calibration parameter corrections $\mathrm{d} k$ and $\mathrm{d} P$ are solutions of the least-squares method of adjustment. We use an iterative procedure, by introducing computed corrections for a new adjustment, until the convergence of calibration parameters is achieved. We achieve the iterative procedure when corrections become lower than $10^{-9}$ arcsecond for both coordinates. Generally, the convergence occurred after no more than three iterations.

\subsection{Comparison of calibration parameters values}

Calibration parameters for each dataset of observations presented in Table 1 have been computed from the CCD reduction method described in the subsection above. Different values were obtained with the various theories used. Calibration parameters are supposed to be constant for each dataset, as the CCD cell was not moved from the telescope. We have successively computed the values of calibration parameters from the four contemporary theories. These values are given in Cols. $2-5$ of Table 1 . For comparison, the sixth column indicates values previously adopted by Harper et al. $(1997,1999)$ and by Qiao et al. (1999) from their own respective methods. In these papers, Qiao derived calibration parameters from the multitheory method including the four contemporary theories and Harper used his own theory (Harper \& Taylor 1993). Table 1 shows that the values of the calibration parameters we have computed for Harper's observations (Col. 3) are slightly different to the values he had previously adopted (Col. 6). This can be explained as we have used in this paper the newest version of this theory (Harper \& Taylor 1999).

In order to extend the comparison of our calibration parameter values with other ones, we have determined for the particular Q961019 dataset of Table 1, made on the 19th of October 1996, the scale and the position angle of the CCD frame from other methods presented above in the introduction. We have successively used the double star 61 Cygni, the globular cluster M 15, and the multi-theory method. The values obtained are presented in Table 2. Significant discrepancies can be observed between the values derived from these different methods. Values obtained from the globular cluster M 15 and the multi-theory method are in good agreement, but they appear significantly different from those obtained from the double star 61 Cygni.

In Table 1, we can also observe discrepancies between the values of the calibration parameters that we derived from the different four contemporary theories, however, they appear much smaller than those obtained from previous methods, as shown in Table 2.

In addition, we have determined that a difference between two scales $\delta k$ of $10^{-4 \prime \prime} /$ pixel, generally observed in Table 1, can lead to differences in the derived satellite positions of about $0^{\prime \prime} 1$. However, we need satellite positions with a greater accuracy in order to improve analytical theories, particularly TASS. Mutual events are of great
Table 2. Calibration parameters $k$ and $\delta P$ obtained from different CCD astrometric reduction methods for the dataset Q961019 of Table 1

\begin{tabular}{lll}
\hline Method & $\delta P(\circ)$ & $k\left(^{\prime \prime} /\right.$ pixel $)$ \\
\hline Double stars $(1)$ & 7.5782 & 0.250522 \\
Double stars(2) & 7.4654 & 0.251103 \\
Globular cluster M 15 & 7.4782 & 0.251161 \\
Multi-theory & 7.4782 & 0.251275 \\
\hline
\end{tabular}

(1) 3 images of 61 Cygni used.

(2) 10 images of 61 Cygni used.

interest for this purpose (Thuillot et al. 2000) but they are unfortunately very rare. Because photographic plates are no longer manufactured, the recent CCD observation technique could be the solution of the future. However, the astrometric reduction used in this technique must be improved. This is one of the special aims of this paper.

As a preliminary analysis of calibration parameters, given in Tables 1 and 2, CCD reduction methods using satellite theories seem to give more coherent values than other methods using reference stars. This can be explained, as these stars are not located in the field of the observed satellites. Moving the telescope may cause flexions and then significant changes in calibration parameters (Colas \& Arlot 1991). Because CCD target are too small to allow reduction from field stars, we prefer CCD reduction methods using satellite theories. To improve these methods, we will compare the four available theories, so as to discover if some, more accurate than others, could be used in preference to any other one.

\section{Comparison of satellite theories}

\subsection{Analysis of residuals for all satellites}

Using the iterative procedure to determine the calibration parameters described above, we computed observedminus-computed residuals from intersatellite positions, in separation $(\Delta S)$ and position angle $(S \Delta P)$, both expressed in arcseconds. These residuals are given in Table 3 for each theory and for each satellite, Titan excepted, as it was used as a reference. Residuals were calculated from satellite observed positions obtained from CCD reduction using the theory mentioned in the first line of Table 3, compared to computed positions obtained from the same theory. Residuals higher than $00^{\prime \prime} 8$ were eliminated. A preliminary analysis of Table 3 shows the very high quality of the ephemeris of satellites Tethys, Dione and Rhea, with residuals lower than $0^{\prime \prime} 1$, whichever theory used. Titan, used as the reference, also can be considered as one of the satellites with the best-known theory. Enceladus presents higher residuals, from $0{ }^{\prime \prime} 1$ to $0^{\prime \prime} 2$. Mimas, Hyperion and Iapetus have much less accurate theories, with residuals between $00^{\prime \prime} 1$ and 0.5 . 
Table 3. Statistics of observed-minus-computed residuals, expressed in arcseconds, for all observations of Table 1, for each theory and for each satellite, Titan excepted (used as the reference satellite). The numbers of observed coordinates used are in parenthesis

Table 3.1. Residuals in separation $\Delta S$

\begin{tabular}{lllll}
\hline & T\&S & H\&T & TASS & Dourneau \\
\hline Mimas & $.3127(54)$ & $.2465(58)$ & $.2716(57)$ & $.2857(58)$ \\
Enceladus & $.2054(204)$ & $.1666(197)$ & $.1795(199)$ & $.1704(197)$ \\
Tethys & $.0905(413)$ & $.0746(413)$ & $.0722(413)$ & $.0814(413)$ \\
Dione & $.0967(438)$ & $.0800(438)$ & $.0750(438)$ & $.0865(438)$ \\
Rhea & $.0864(503)$ & $.0702(503)$ & $.0679(503)$ & $.0784(503)$ \\
Hyperion & $.3538(135)$ & $.2492(218)$ & $.1227(218)$ & $.4266(141)$ \\
Iapetus & $.3076(233)$ & $.1493(230)$ & $.1081(230)$ & $.1864(230)$ \\
\hline
\end{tabular}

Table 3.2. Residuals in position angle $S \Delta P$

\begin{tabular}{lllll}
\hline & T\&S & H\&T & TASS & Dourneau \\
\hline Mimas & $.3220(54)$ & $.1508(58)$ & $.1508(57)$ & $.1644(58)$ \\
Enceladus & $.1404(204)$ & $.1100(197)$ & $.1286(199)$ & $.1312(197)$ \\
Tethys & $.0803(413)$ & $.0728(413)$ & $.0717(413)$ & $.0877(413)$ \\
Dione & $.0699(438)$ & $.0694(438)$ & $.0678(438)$ & $.0734(438)$ \\
Rhea & $.0960(503)$ & $.0832(503)$ & $.0828(503)$ & $.0957(503)$ \\
Hyperion & $.3877(135)$ & $.1660(218)$ & $.1979(218)$ & $.5205(141)$ \\
Iapetus & $.5302(223)$ & $.2378(230)$ & $.2064(230)$ & $.2252(231)$ \\
\hline
\end{tabular}

Table 3 shows some significant discrepancies between residuals obtained from each different theory. TASS is the theory which generally gives the smallest residuals for satellites with the best-known theories, with values from 0 ".07 to 0 '.08. However, for these satellites, all other theories also give very satisfactory residuals, from $0 . \prime 07$ to 0 .'1 and the differences with TASS appear quite small. For other satellites, TASS is not always the best theory. Harper \& Taylor's (1999) theory generally gives the best residuals for Mimas and Enceladus. Dourneau's theory also gave better residuals in separation than TASS for Enceladus. For Iapetus, TASS presents the best residuals, with 0 !! 1 in $\Delta S$ and 0 !' 2 in $S \Delta P$.

Thus, as a general result of the analysis of residuals presented in Table 3, TASS can be considered as the best theory. Both the theory developed by Harper \& Taylor and by Dourneau are rather close and can be placed in second position after TASS, with a slight advantage for Harper \& Taylor, especially for Hyperion. Such a classification is not surprising, as we have seen in Sect. 2 above that Dourneau's and Harper \& Taylor's theories were built from very similar adjustments for all satellites, Hyperion excepted. For this satellite, the residuals from Dourneau are the highest $(0.5)$, but from Harper \& Taylor they are very close to the lowest ones from TASS $\left(00^{\prime \prime} 2\right)$. This can be
Table 4. Statistics of $\mathrm{O}-\mathrm{C}$ residuals $\Delta S$ and $S \Delta P$ of calibration satellites Tethys, Dione, Rhea and Titan, used as a reference satellite, for all observations presented in Table 1 and for each contemporary theory. The numbers of observed coordinates used are in parenthesis

\begin{tabular}{lcccc}
\hline Theory & TASS & H\&T & Dourneau & T\&S \\
\hline$\Delta S(1359)$ & 0.0715 & 0.0748 & 0.0819 & 0.0910 \\
$S \Delta P(1355)$ & 0.0749 & 0.0759 & 0.0846 & 0.0835 \\
\hline
\end{tabular}

explained as Dourneau did not include short-period terms in the expressions for Hyperion, and we have used in this paper the new version (Harper \& Taylor 1999) derived from the theory improved by Taylor (1992). But we have noted above that discrepancies between residuals obtained from each of the four theories remain very small for satellites with the best-known theories. Then, for these satellites, it seems very difficult to appreciate the respective quality of TASS, Harper \& Taylor and Dourneau theories. The comparison of their respective residuals in Table 3 is generally under the 0. . 01 level and any of these theories can reach such a precision. But Table 3 clearly shows that Taylor \& Shen's theory results in higher residuals than all the other theories for all satellites and especially for Mimas and Iapetus ( $0 . .3$ to $0 . .5)$.

In conclusion, this analysis has shown that all four contemporary theories analyzed in this paper are adequate to represent accurate orbits of Tethys, Dione, Rhea and Titan. Thus, these satellites can reasonably be used as calibration satellites. For other satellites, we can conclude that ephemerides from all theories still need to be improved, especially for Taylor \& Shen and also, in the particular case of Hyperion, for Dourneau.

\subsection{Comparison of residuals for calibration satellites}

Now, we propose to compare the residuals for all the observations of the calibration satellites given in Table 1, relative to each theory. A few low-quality observed positions with residuals greater than $3 \sigma$ were eliminated. The residuals obtained are presented in Table 4 . They confirm the previous analysis of Table 3. TASS appears again as the best theory in both coordinates for calibration satellites, with very low values, between $00^{\prime \prime} 07$ and 0 .'08. For these satellites, Table 4 also confirms that the three other theories present very satisfactory accuracy, whichever theory was used, with residuals between 0 !'07 and 0 !'09, hardly higher than those from TASS. Harper \& Taylor's theory appears here very close to TASS. All four theories of calibration satellites seem to have about equivalent accuracy (of $0.08^{\prime \prime}$, within only $0.02^{\prime \prime}$ ). Thus, this last value of 0 '.02 could be considered as a preliminary evaluation of the consistency of CCD astrometric reduction methods using any of these four theories of motion. Further, in this paper, we shall try to evaluate more precisely such consistency using different methods. 
Table 5. Residuals between satellite observed positions, reduced from the four theories and from the multi-theory method, and computed positions derived from these four theories. Residuals are computed for all observations of calibration satellites given in Table 1 and are expressed in arcseconds

Table 5.1. Residuals in separation $\Delta S$

\begin{tabular}{|c|c|c|c|c|}
\hline \multirow[t]{2}{*}{$\begin{array}{l}\text { Reduction } \\
\text { method }\end{array}$} & \multicolumn{4}{|c|}{$\begin{array}{c}\text { Computation } \\
\text { theories }\end{array}$} \\
\hline & TASS & $\mathrm{H} \& \mathrm{~T}$ & Dourneau & $\mathrm{T} \& \mathrm{~S}$ \\
\hline TASS & 0.0715 & 0.0840 & 0.0769 & 0.0923 \\
\hline $\mathrm{H} \& \mathrm{~T}$ & 0.0865 & 0.0748 & 0.0851 & 0.1131 \\
\hline Dourneau & 0.0871 & 0.0915 & 0.0819 & 0.1137 \\
\hline $\mathrm{T} \& \mathrm{~S}$ & 0.1082 & 0.1245 & 0.1205 & 0.0910 \\
\hline Multi-theory & 0.0728 & 0.0871 & 0.0879 & 0.1030 \\
\hline \multicolumn{5}{|c|}{ Table 5.2. Residuals in position angle $S \Delta P$} \\
\hline \multirow[t]{2}{*}{$\begin{array}{l}\text { Reduction } \\
\text { method }\end{array}$} & \multicolumn{4}{|c|}{$\begin{array}{c}\text { Computation } \\
\text { theories }\end{array}$} \\
\hline & TASS & $\mathrm{H} \& \mathrm{~T}$ & Dourneau & $\mathrm{T} \& \mathrm{~S}$ \\
\hline TASS & 0.0749 & 0.0762 & 0.0873 & 0.0843 \\
\hline $\mathrm{H} \& \mathrm{~T}$ & 0.0762 & 0.0759 & 0.0881 & 0.0847 \\
\hline Dourneau & 0.0845 & 0.0850 & 0.0846 & 0.0972 \\
\hline $\mathrm{T} \& \mathrm{~S}$ & 0.0833 & 0.0837 & 0.1015 & 0.0835 \\
\hline Multi-theory & 0.0751 & 0.0757 & 0.0833 & 0.0831 \\
\hline
\end{tabular}

\section{Comparison of reduction methods using different theories}

\subsection{Analysis of residuals depending on the reduction made from different theories}

Now, we propose to analyze and compare various CCD reduction methods using different satellite theories. First, we will try to point out the possible bias that any theory used in the reduction can introduce in the derived satellite positions. So, for all observations of calibration satellites presented in Table 1, we determined the five different positions, successively obtained from the reduction using the four theories and from the multi-theory method. Then, we computed residuals by comparing these five observed positions to positions computed from each theory. Table 5 presents the derived residuals. We can see that particular residuals in the diagonal of Table 5, obtained from the comparison of satellite observed positions to computed positions, derived using the same theory, were already given in Table 4 . It is interesting to note in Table 5 that diagonal residuals generally appear as the smallest ones. This points out a significant bias of theories towards corresponding satellite positions affected with systematic errors. Future adjustment of theories could be affected if such biased satellite positions are used.

\subsection{Analysis of differences between observations reduced from different theories}

We shall try to evaluate the bias pointed out above. We have determined, for each satellite, differences between the four successive positions, obtained from each theory in the CCD reduction, and positions derived from the multitheory method, used as a reference method, described above in the introduction. These differences, computed for each night of observation listed in Table 1, are visualized in Figs. 1 and 2 for both coordinates, separation $\Delta S$ and position angle $S \Delta P$, respectively.

From a preliminary analysis, all differences appearing in Figs. 1 and 2 are lower than $0{ }^{\prime \prime} 1$, with an exception for some rare points relative to Taylor \& Shen's theory. Most differences remain lower than $00^{\prime \prime} 02$, whatever the theory used. This confirms our preliminary evaluation of the consistency of reduction methods using satellite theories obtained above from residuals of Table 4. However, some exceptions appear, especially in Fig. 1. If observed positions successively reduced from the four theories present a rather good coherency in position angle, generally within 0.02 (cf. Fig. 2), they can present some higher discrepancies in separation. For this coordinate, Fig. 1 emphasizes differences between $00^{\prime \prime} 05$ and $0{ }^{\prime \prime} 1$, especially for Harper \& Taylor and Taylor \& Shen's theories. In addition, it is interesting to note that these differences generally are positive for Harper \& Taylor and negative for Taylor \& Shen, pointing out a possible systematic bias in satellite observed positions reduced from both of these theories. Such a bias can be confirmed from the analysis of Table 6 which presents mean values of differences visualized in Figs. 1 and 2, with their root mean square (rms) errors, for all observations of each satellite. Table 6 shows that mean values are always positive for Harper \& Taylor and negative for Taylor \& Shen, confirming the bias relative to these theories pointed out in Fig. 1. One of the highest bias concerns Harper \& Taylor's theory, with about 0.03 in separation. It may be related to possible systematic scale errors in the observations, already mentioned by Harper \& Taylor (1993). In addition, Table 6 shows that for each satellite, TASS generally presents the lowest mean differences, under $0{ }^{\prime \prime} 005$, and the lowest rms errors, under $0 . \prime 02$. This means that observed positions reduced from TASS are the most coherent with those reduced from the multi-theory method. As this method was proposed by Qiao et al. (1999) for reducing systematic errors of each theory in satellite positions, we can expect that positions reduced from TASS would also have no significant systematic errors.

To further evaluate the accuracy of satellite observations reduced from different theories, we propose to use rms errors of mean differences given in Table 6 , generally higher than the corresponding mean differences. These errors are lowest for TASS. The CCD reduction using this theory should then provide the most consistent satellite observations, as rms errors in Table 6 are under 0.'015 in both coordinates. Thus, for TASS, this last value appears 

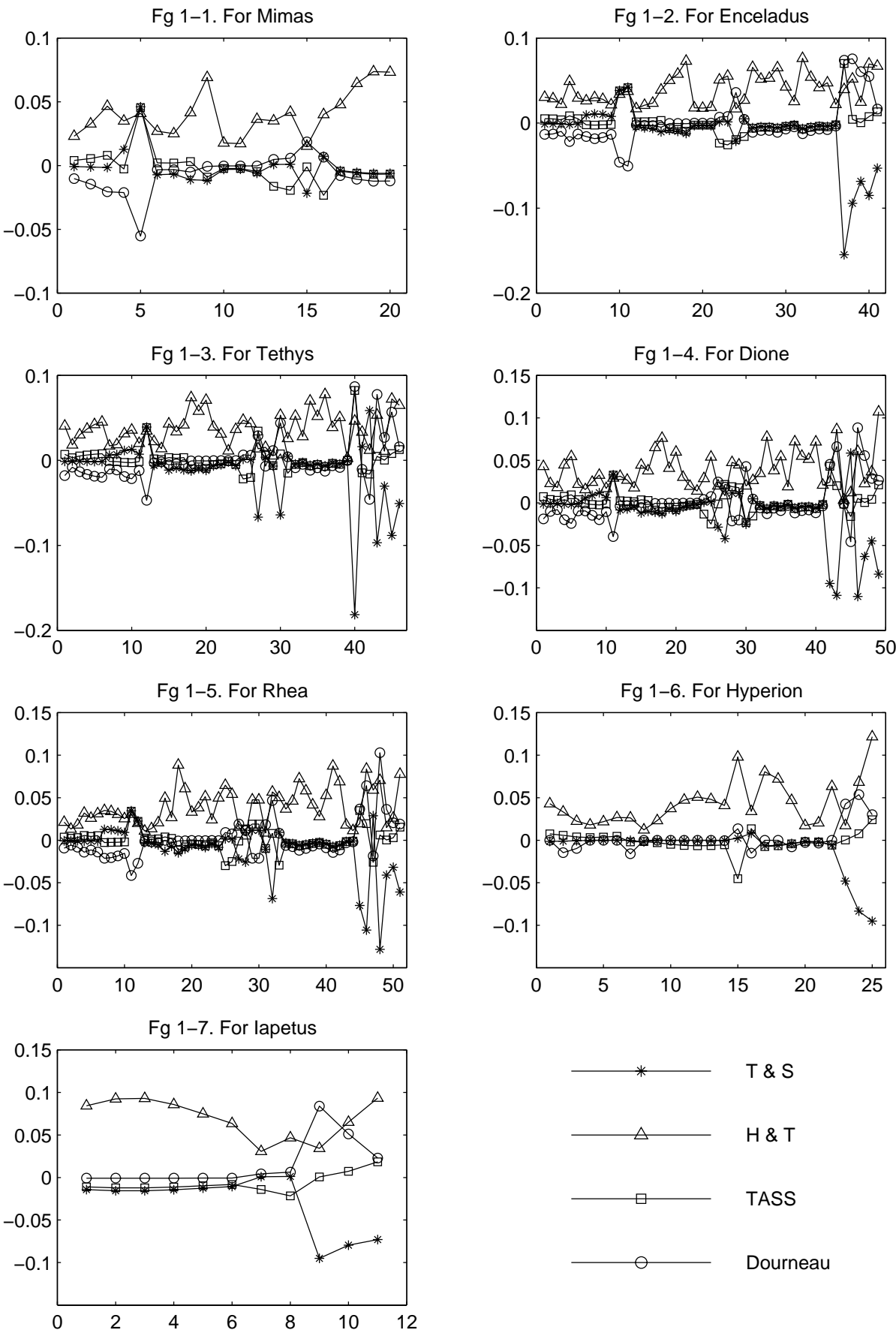

Fig. 1. Differences in separation $\Delta S$ between satellite positions reduced from the four successive theories and from the multitheory method. Ordinates are in arcseconds. Abscissas give the sequence of nights of observation

somewhat better than our preliminary evaluation $\left(0{ }^{\prime \prime} 02\right)$ concerning reduction methods involving any satellite theory. However, when theories other than TASS are used in the reduction, the accuracy of observations, also evaluated from rms errors of Table 6, appears significantly higher: about 0 .'03 for Harper \& Taylor and Dourneau, and 0. ".04 for Taylor \& Shen.

\section{Conclusion}

We have analysed the four contemporary satellite theories developed by Taylor \& Shen (1988), Dourneau (1993), Duriez \& Vienne (1997) and Harper \& Taylor (1999), to get some information about their respective reliability. We have shown that these four theories can derive quite 

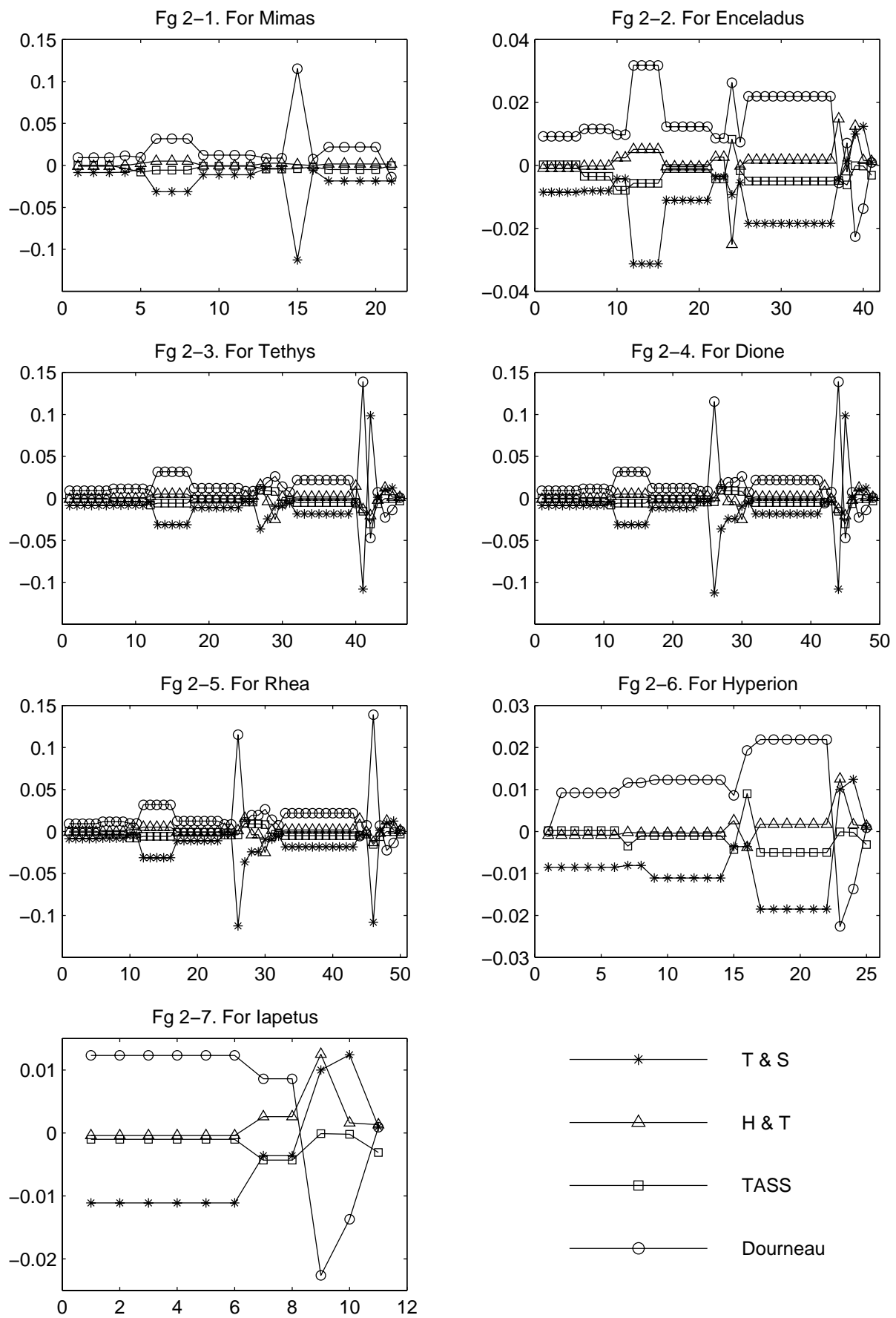

Fig. 2. Differences in position angle $S \Delta P$ between satellite positions reduced from the four successive theories and from the multi-theory method. Ordinates are in arcseconds. Abscissas give the sequence of nights of observation

satisfactory orbits for Tethys, Dione, Rhea and Titan, which can be used as calibration satellites for computing calibration parameters in CCD reduction. For these satellites, whichever theory used in the CCD reduction, we can expect to obtain observed positions with a good accuracy. However, we have pointed out a bias of the theories used in the CCD reduction in the corresponding derived satellite positions, rather significant for Harper \& Taylor and Taylor \& Shen, with values of about 0.'03. In order to limit systematic errors, especially those introduced when only one theory is used in the reduction process, we had proposed in a previous work (Qiao et al. 1999) the multi-theory reduction method using all four contemporary theories together. In this paper, we have shown that the CCD reduction using the TASS theory is in better agreement with the multi-theory method than all other reduction methods. So, when used in the CCD reduction, TASS should not introduce any significant bias in the satellite positions. Also, we have made some attempts to evaluate the accuracy of satellite positions reduced from 
Table 6. Mean differences in satellite observations depending on the reduction made, for all observations presented in Table 1. These differences compare positions reduced from each theory to the corresponding positions reduced from the multi-theory method. Mean differences are affected with rms errors computed for all observations, and given in separation $\Delta S(\mathrm{~S})$ and position angle $S \Delta P(\mathrm{P})$. All values are given in milli-arcsecond

\begin{tabular}{|c|c|c|c|c|c|}
\hline Satellite & & $T \& S$ & $\mathrm{H} \& \mathrm{~T}$ & TASS & Dourneau \\
\hline \multirow[t]{2}{*}{ Mimas } & (S) & $-5.5 \pm 11.6$ & $36.0 \pm 17.0$ & $-1.2 \pm 10.7$ & $3.5 \pm 15.1$ \\
\hline & $(\mathrm{P})$ & $-32.4 \pm 40.7$ & $0.9 \pm 1.9$ & $-2.6 \pm 2.3$ & $33.1 \pm 40.6$ \\
\hline \multirow[t]{2}{*}{ Enceladus } & $(\mathrm{S})$ & $-34.8 \pm 50.2$ & $38.8 \pm 17.1$ & $4.7 \pm 24.1$ & $18.5 \pm 34.0$ \\
\hline & $(\mathrm{P})$ & $-6.6 \pm 11.3$ & $2.2 \pm 9.0$ & $-2.0 \pm 3.5$ & $6.5 \pm 16.0$ \\
\hline \multirow[t]{2}{*}{ Tethys } & $(\mathrm{S})$ & $-34.9 \pm 42.7$ & $39.1 \pm 24.9$ & $4.1 \pm 15.5$ & 29.6 \\
\hline & $(\mathrm{P})$ & $-0.5 \pm 23.7$ & $2.7 \pm 8.9$ & $-1.9 \pm 6.3$ & $-0.2 \pm 22.3$ \\
\hline \multirow[t]{2}{*}{ Dione } & $(\mathrm{S})$ & $-32.6 \pm 38.4$ & $39.2 \pm 27.3$ & $5.1 \pm 8.9$ & $16.6 \pm 28.8$ \\
\hline & (P) & $-7.3 \pm 29.1$ & $2.1 \pm 9.0$ & $-1.4 \pm 7.2$ & $6.8 \pm 28.0$ \\
\hline \multirow[t]{2}{*}{ Rhea } & $(\mathrm{S})$ & $-25.0 \pm 30.8$ & $33.3 \pm 23.4$ & $2.5 \pm 12.8$ & $13.5 \pm 24.7$ \\
\hline & (P) & $-11.8 \pm 27.2$ & $3.4 \pm 8.2$ & $-0.7 \pm 5.0$ & $=31.0$ \\
\hline \multirow[t]{2}{*}{ Hyperion } & $(\mathrm{S})$ & $-44.6 \pm 33.5$ & $40.0 \pm 32.8$ & $-3.4 \pm 8.8$ & $29.1 \pm 23.3$ \\
\hline & (P) & $-0.3 \pm 11.5$ & $4.7 \pm 6.0$ & $-0.7 \pm 2.7$ & $-4.1 \pm 17.6$ \\
\hline \multirow[t]{2}{*}{ Iapetus } & (S) & $-70.8 \pm 31.7$ & $57.4 \pm 22.9$ & $2.6 \pm 10.0$ & $49.8 \pm 31.1$ \\
\hline & (P) & $6.1 \pm 7.7$ & $5.4 \pm 5.4$ & $-1.0 \pm 1.4$ & $-10.3 \pm 12.6$ \\
\hline
\end{tabular}

the multi-theory method and from CCD reduction using the four successive contemporary theories. From the CCD observed data from 1990 to 1997 analyzed in this paper, we have shown that, for calibration satellites, observations reduced from TASS seem to present the best consistency $\left(0^{\prime \prime} 015\right)$, while those reduced from Harper \& Taylor and Dourneau generally have a consistency of 0.03 or better, and Taylor \& Shen of about 0 !'05.

In conclusion, the analysis we have performed has shown that the multi-theory method appears to be useful in CCD reduction, owing to its ability to provide satellite observations without any significant systematic error. However, such satellite observations reduced from the multi-theory method can be affected by the significant errors introduced by the less accurate theories such as Taylor \& Shen. So, we suggest the use of the highly consistent TASS theory alone for computing calibration parameters in CCD reduction. The observations should then have a consistency of about $0{ }^{\prime} 015$ and they should not present any significant systematic errors. Our suggestions about CCD reduction methods should help observers to publish in the future more accurate reduced positions of Saturn's satellites. However, we advise them to publish in addition all their raw data, measured in pixels on the CCD frame. This will allow subsequent users of the satellite observations to adopt a given method of reduction, as these methods should be improved in the near future, particularly when larger CCD targets will be used. For instance, wider fields will allow classical reduction from reference field stars. This will resolve many model-dependant systematic errors from future positions of Saturn's satellites.

Acknowledgements. We wish to thank Prof. B. C. Qiao and his staff at the Sheshan Station of Shanghai Observatory. Also, we thank the staff at the Yunnan Astrometrical Observatory for their help during observations. We are grateful to Dr. D. Harper for providing us with the new version of satellite theories, as well as to Dr. D. B. Taylor for his newest version of Hyperion's theory. This work was carried out with the financial support of the National Science Foundation of China (NSFC).

\section{References}

Beurle, K., Harper, D., Jones, D. H. P., et al. 1993, A\&A, 269, 564

Colas, F., \& Arlot, J. E. 1991, A\&A, 252, 402

Dourneau, G. 1987, Observation et étude du mouvement des huit premiers satellites de Saturne, Thèse de doctorat d'État, Université Bordeaux I

Dourneau, G. 1993, A\&A, 267, 292

Dourneau, G., \& Baratchart, S. 1999, A\&A, 350, 680

Duriez, L., \& Vienne, A. 1991, A\&A, 243, 263

Duriez, L., \& Vienne, A. 1997, A\&A, 324, 366

Harper, D., \& Taylor, D. B. 1993, A\&A, 268, 326

Harper, D., Murray, C. D., Beurle, K., et al. 1997, A\&A, 121, 65

Harper, D., Beurle, K., Williams, I. P., et al. 1999, A\&AS, 136, 257

Harper, D., \& Taylor, D. B., 1999, personal communication

Jones, D. H. P., Sinclair, A. T., \& Williams, I. P. 1989, MNRAS, 237, 15P

Pascu, D. 1982, data held at the U.S. Naval Observatory, Washington, DC

Pascu, D., Seidelman, P. K., Schmidt, R. S., Santoto, E. J., \& Hershey, J. L. 1987, AJ, 93, 963

Qiao, R. C., Shen, K. X., Liu, J. R., \& Harper, D. 1999, A\&AS, 137,1

Shen, K. X., \& Qiao, R. C. 1996, Proceeding of the 172nd Symposium of the IAU, 141

Strugnell, P. R., \& Taylor, D. B. 1990, A\&AS, 83, 289

Taylor, D. B., \& Shen, K. X. 1988, A\&A, 200, 269

Taylor, D. B. 1992, A\&A, 265, 825

Thuillot, W., Arlot, J. E., Ruatti, C., et al. 2000, A\&A, to be published

Vienne, A., \& Duriez, L. 1991, A\&A, 246, 619

Vienne, A., \& Duriez, L. 1992, A\&A, 257, 351

Vienne, A., \& Duriez, L. 1995, A\&A, 297, 588

Woltjer, J. 1928, Ann. Sterw. Leiden., XVI, 3 